\title{
Extensions of PDZ domains as important structural and functional elements
}

\author{
Conan K. Wang ${ }^{1,2}$, Lifeng Pan ${ }^{1}$, Jia Chen ${ }^{1}$, Mingjie Zhang ${ }^{1 凶}$ \\ ${ }^{1}$ Department of Biochemistry, Molecular Neuroscience Center, Hong Kong University of Science and Technology, Clear Water \\ Bay, Kowloon, Hong Kong \\ 2 Eskitis Institute for Cell and Molecular Therapies, Griffith University, Brisbane, QId 4111, Australia \\ 凶 Correspondence: mzhang@ust.hk \\ Received July 17, 2010 Accepted July 21, 2010
}

\begin{abstract}
'Divide and conquer' has been the guiding strategy for the study of protein structure and function. Proteins are divided into domains with each domain having a canonical structural definition depending on its type. In this review, we push forward with the interesting observation that many domains have regions outside of their canonical definition that affect their structure and function; we call these regions 'extensions'. We focus on the highly abundant PDZ (PSD-95, DLG1 and ZO-1) domain. Using bioinformatics, we find that many PDZ domains have potential extensions and we developed an openly-accessible website to display our results (http:/l bcz102.ust.hk/pdzex/). We propose, using well-studied PDZ domains as illustrative examples, that the roles of PDZ extensions can be classified into at least four categories: 1) protein dynamics-based modulation of target binding affinity, 2) provision of binding sites for macro-molecular assembly, 3) structural integration of multi-domain modules, and 4) expansion of the target ligand-binding pocket. Our review highlights the potential structural and functional importance of domain extensions, highlighting the significance of looking beyond the canonical boundaries of protein domains in general.
\end{abstract}

\section{KEYWORDS PDZ domain, PDZ extensions, protein structure}

\section{INTRODUCTION}

A central paradigm within structural biology is the concept of domains (Pawson and Nash, 2003). In past definitions, domains were thought of as units of compact structure, evolution and folding, and/or function. With the advent of modern bioinformatics, the conservation of domains throughout evolution became instantly recognizable, leading to the established view that domains can be represented by a specific pattern of secondary structure elements that adopt a canonical form when in solution (Schultz et al., 1998; Bateman et al., 2004). It is much less appreciated but still important that a significant number of domains have additional elements of structure that lie almost immediately before or after the canonical domain, extending the domain. The presence of these extensions and their impact on folding, structure, dynamics and function of the domain to which they are attached is of particular significance for PDZ domains (which was based on three proteins-PSD-95, DLG1 and ZO-1 - that led to its discovery) due to the abundance and prevalence of the associated structured extensions.

Since their discovery, the PDZ domains have quickly become one of the most abundant and widely distributed (found in humans, plants, insects, yeast and even bacteria) known structural domains (Harris and Lim, 2001; Zhang and Wang, 2003), making them an excellent model for examining the role of domain extensions. Although some PDZ domains were recently shown to have dynamic functions (Mishra et al., 2007), they generally act as scaffolds, helping to assemble large molecular complexes (Zhang and Wang, 2003; Kim and Sheng, 2004; Feng and Zhang, 2009). The archetypical PDZcontaining protein, PSD-95, is the most abundant scaffold protein in the post-synaptic density (PSD), and is believed to interact with a large number of other proteins within the PSD, including NMDA receptor $K^{+}$channels, neuronal nitric oxide synthase (nNOS), and the cytoskeletal protein cysteine-rich PDZ binding protein (CRIPT) (Kim et al., 1995; Kornau et al., 1995; Brenman et al., 1996a; Niethammer et al., 1998). The presence of PDZ proteins, such as the ones shown in Fig. $1 \mathrm{~A}$, in many important cellular processes, combined with the 
potential of PDZ proteins as therapeutic targets (Blazer and Neubig, 2009) justifies the significant and ongoing scientific interest in the PDZ domain.

Structural biology has contributed to our understanding of the mechanism of action of PDZ domains. The canonical PDZ domain, which is depicted in Fig. 1B, consists of six $\beta$-strands and two $\alpha$-helices, and folds into a compact structure that uses $\beta B$ and $\alpha B$ to form a conserved binding groove. It is surprising and worth noting that the first structure of a PDZ domain (the third PDZ of PSD95) contained an additional Cterminal helix (Doyle et al., 1996); however, the functional importance of the extension was initially overlooked because it was spatially distal from the binding pocket. Although the 'classical' binding mode dictates that PDZ domains bind to the four most C-terminal residues of a particular target, there are cases where PDZ domains can bind to internal peptide regions or segments longer than four residues (Stiffler et al., 2007; Tonikian et al., 2008). The region of the target that interacts with PDZ is more commonly referred to as the PDZ binding motif (PBM). Many PDZ domains are also known to bind specific lipids (Zimmermann et al., 2002; Mortier et al., 2005; Yan et al., 2005; Wu et al., 2007).

It is not surprising that the affinity of a PDZ domain for a peptide target is sensitive to both the composition of residues within the binding pocket of the PDZ domain and the PBM of the target. Furthermore, it is well-documented that the binding affinity can also depend on the phosphorylation state of the target (Cohen et al., 1998; Chung et al., 2000; Hegedüs et al., 2003), as well as the conditions of the surrounding solution, such as the ionic strength, $\mathrm{pH}$, and redox potential (Chi et al., 2006; Mishra et al., 2007). It was surprising when studies suggested that, in the canonical PDZ domain, residues away from the binding pocket can affect the binding affinity (Lockless and Ranganathan, 1999), because such residues were previously thought to have only a supporting structural role. Perhaps this finding provided an early indication that extensions, which lie away from the binding groove, may also be potentially important (for binding at least). Emerging evidence suggest that extension sequences of PDZ domains are likely to have specific structural and/or functional roles.

To assess how many PDZ domains might be affected by extensions, we performed a bioinformatics-based search for extended PDZ domains (the results of which will be presented herein), and found a significant number of domains that are potentially extended. This immediately raises the question: why do these extensions exist? Here, we propose that the roles of these extensions can be classified into categories depending on the functional and structural association of the extension with the core domain.

\section{EXTENDED PDZ DOMAINS ARE HIGHLY PREVALENT}

We sought to identify the prevalence of extended PDZ domains using bioinformatics. We began with the simple definition that extensions are regular structured segments that lie outside the canonical domain boundary. Our overall strategy was to predict, using computational programs,
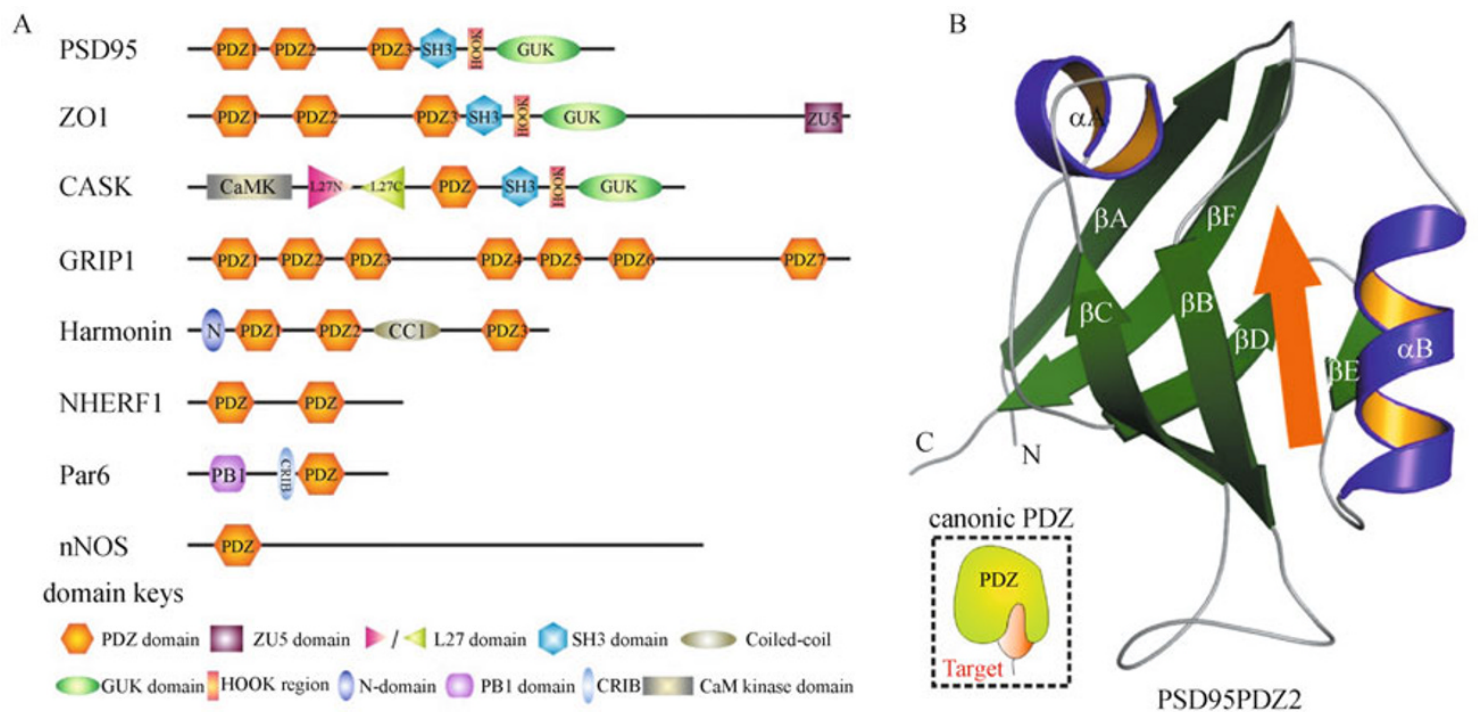

Figure 1. PDZ domains proteins and the canonical form of a PDZ domain. Panel A shows the domain architecture of selected PDZ-containing proteins. A key describing the different types of domains within these proteins is shown at the bottom of the panel. Panel B shows a ribbon representation of the structure of the second PDZ (PDZ2) domain of PSD-95 (PDB ID: 1QLC). PSD-95 PDZ2 contains six $\beta$-strands and two $\alpha$-helices (labeled), and adopts the canonical PDZ fold that displays a binding groove for a peptide ligand (shown as an orange arrow). The inset is a cartoon representation of the typical PDZ-ligand interaction. 
whether regions outside known PDZ domains contain structure. We have collated the results into a publiclyaccessible website (http://bcz102.ust.hk/pdzex/) that contains tools for searching and data visualization, enabling a user to easily browse and review predictions of extended PDZ domains. We also provide a more detailed description of our methodology used to generate the predictions on the website.

Briefly, PDZ-containing protein sequences and their associated domain boundary definitions were extracted from UniProt, a source of curated protein entries, because the quality and accuracy of the annotation was important for this study. The domain boundary definitions from UniProt provided the start and end positions of the canonical PDZ domain within each sequence, from which we examined sequence segments of an arbitrary length (i.e., 50 amino acids) that are immediately upstream or downstream of the canonical domain. As prediction programs can be sensitive to the composition of the input sequence, we made several predictions using different input sequences (see our website for more details) that differed by their start and end positions. As an example, Fig. 2A shows three types of input sequences that were used, i.e., the $\mathrm{N}$-terminal sequence segment only, the C-terminal sequence segment only, and an entire region that includes the $\mathrm{N}$-terminal and $\mathrm{C}$-terminal sequence segment and the central PDZ domain. We used the programs PSIPRED (Jones, 1999), PROFPHD (Rost et al., 2004), and PREDATOR (Frishman and Argos, 1996) to predict secondary structure and DISEMBL (Linding et al., 2003) and DISOPRED (Ward et al., 2004) to predict disorder within the input sequences.

Using the aforementioned approach, we extracted 154 fulllength human PDZ proteins with reviewed annotations from UniProt, giving us a total of 269 separate human PDZ domains. Initially, we precluded extensions from being part of any known domain; however, there is no reason why extensions cannot be part of other domains or be domains themselves (as discussed later). Based on predictions using only the extension sequences as input, we were surprised to find that an alarming number of PDZ domains (i.e., $~ 80 \%$; Fig. 2B) have potential structure in their extensions at the two termini. This figure fluctuated depending on the predictor or type of input used; for example, using input sequences comprising the domain and the extensions on either end, only about $40 \%$ of PDZ domains were predicted to have structured extensions (Fig. 2B). Nonetheless, the prediction results as a whole suggest that there may be a significant number of extended PDZ domains. Further analysis (see website for more details) reveals that structured extensions do not favor a particular end of the canonical domain; and when structured segments exist, they tend to occupy a relatively small portion of our defined extension window and, importantly, tend to sit in close proximity to the canonical domain (Fig. 2C), suggesting that they may pack against the core fold. In all, these results suggest that PDZ extensions are prevalent and potentially significant.

To gain some indication whether PDZ extensions are present in other species, we performed our bioinformatics analysis on 128 mouse PDZ proteins from which 223 PDZ domains were extracted. For each PDZ domain that we analyzed, detailed prediction results, such as the predicted structure of each residue in the input sequence, can be viewed dynamically on our website. In general, the results of the predictions for mouse PDZ proteins show similar trends to the ones mentioned above for human PDZ proteins, suggesting that extensions may have important roles from an evolutionary perspective. Some examples of human PDZ domains that are extended in humans and mice include the third PDZ domain of PSD-95 (PSD-95 PDZ3), the second PDZ domain of $\mathrm{Na}^{+} / \mathrm{H}^{+}$exchange regulatory factor 1 (NHERF1 PDZ2), the PDZ domain of neuronal nitric oxide synthetase (nNOS PDZ), the third PDZ domain of disc large homolog 1 (DLGh1 PDZ3), and the first PDZ domain of harmonin (harmonin PDZ1) -all of which will be discussed in more detail along with other examples in the following sections.

\section{ROLES OF PDZ EXTENSIONS}

We propose that there are at least four general roles of PDZ extensions: 1) protein dynamics-based modulation of target binding affinity, 2) provision of binding sites for macromolecular assembly, 3) structural integration of multi-domain modules, and 4) expansion of the target ligand binding pocket. We will discuss each of these roles using specific cases as examples.

\section{Dynamics-based modulation of binding affinity}

PSD-95, a member of the membrane associated guanylate kinases (MAGUKs) family of scaffold proteins, comprises three PDZ domains, a Src Homology 3 (SH3) domain, and a guanylate kinase-like (GUK) domain, which interact to form molecular complexes in the cellular environment. The underpinning mechanism behind the interaction of the CRIPT PBM with PSD-95 PDZ3 was defined in a landmark study that was the first to not only characterize the fold of the canonical PDZ domain but also the form of the peptide-bound state, showing that for PSD-95 PDZ3, the backbone conformation is rigid and unaffected by ligand binding (Doyle et al., 1996). Interestingly, these structures are also the first of an extended PDZ domain, showing an additional C-terminal alpha-helix that packs up against the core fold in a region distinct from the peptide ligand binding pocket. The structure of the CRIPT-bound form of PDS-95 PDZ3 is shown in Fig. 3A. There is no direct contact between the additional helix and the bound peptide with the closest distance between the two being $\sim 6 \AA$ measured from the side-chain nitrogen of the -3 position 
A

input and analysis overview
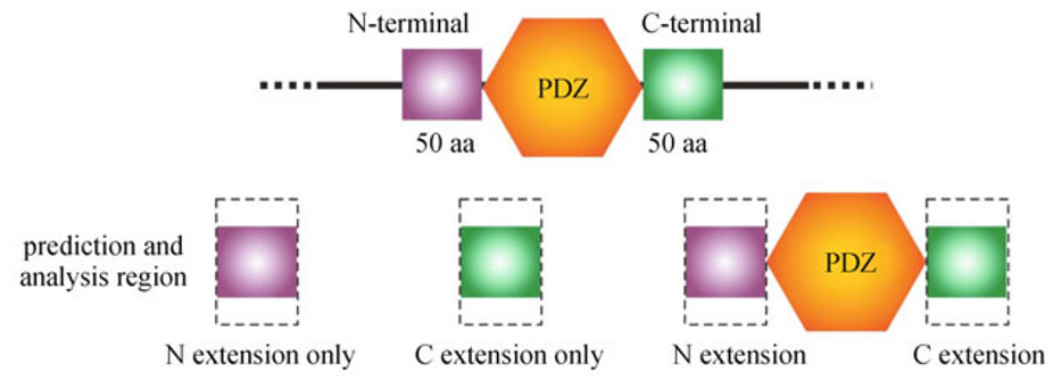

B frequency of structured residues in extensions

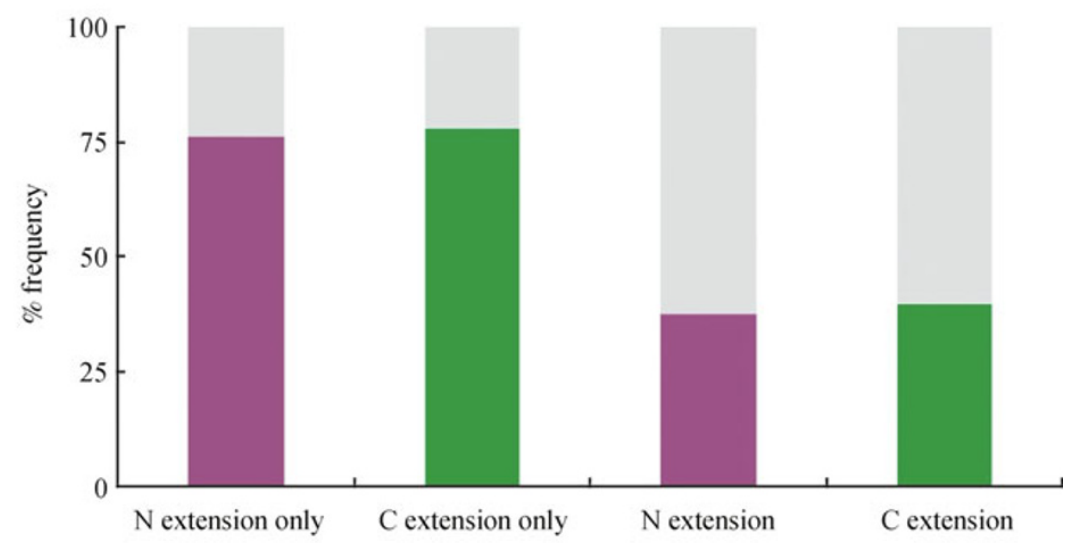

C distance from PDZ boundary to nearest structured residue

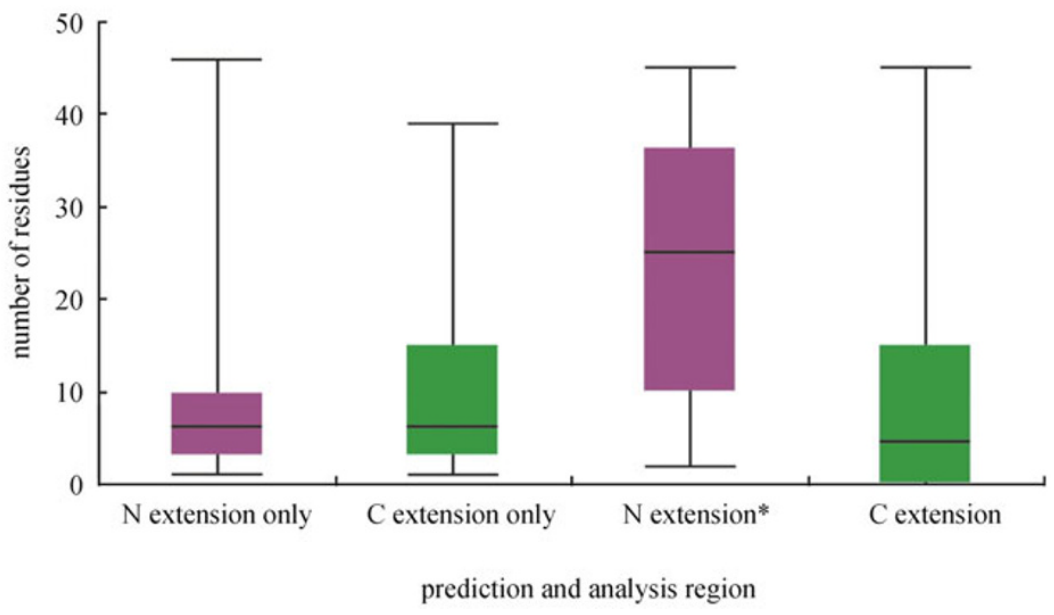

Figure 2. Distribution of extended PDZ domains in humans. Sequences of PDZ domains, and their N-terminal and C-terminal regions of 50 amino acids in length, were extracted from UniProt according to the UniProt sequence annotations. Panel A shows three different types of input sequences that were used for prediction, i.e., $\mathrm{N}$ extension only, $\mathrm{C}$ extension only, and the entire region containing the $\mathrm{N}$ extension, PDZ domain, and C-extension. Regardless of the input sequence, the 50 amino acid sequence segment (enclosed in a dotted box) was analyzed for predicted structure. This figure illustrates the results from using PSIPRED to predict secondary structure. Panel B shows the frequency of extensions with at least one structured residue for each prediction and analysis region. Panel $\mathrm{C}$ shows box plots of the distances (i.e., number of residues) that separate either the N-terminal or C-terminal PDZ domain boundary from the nearest structured residue within the extension for extensions that have predicted structure. The asterisk indicates that the data was post-processed; this was necessary because the length of the PDZ $\beta A$ was often predicted to cross over the defined $\mathrm{N}$-terminal boundary, and so these $\beta$-sheet predictions were discarded from the analysis. For a more detailed description of the complete procedure, including input types and programs used, and a more detailed analysis of the results, please see our website (http://bcz102.ust.hk/pdzex/). 

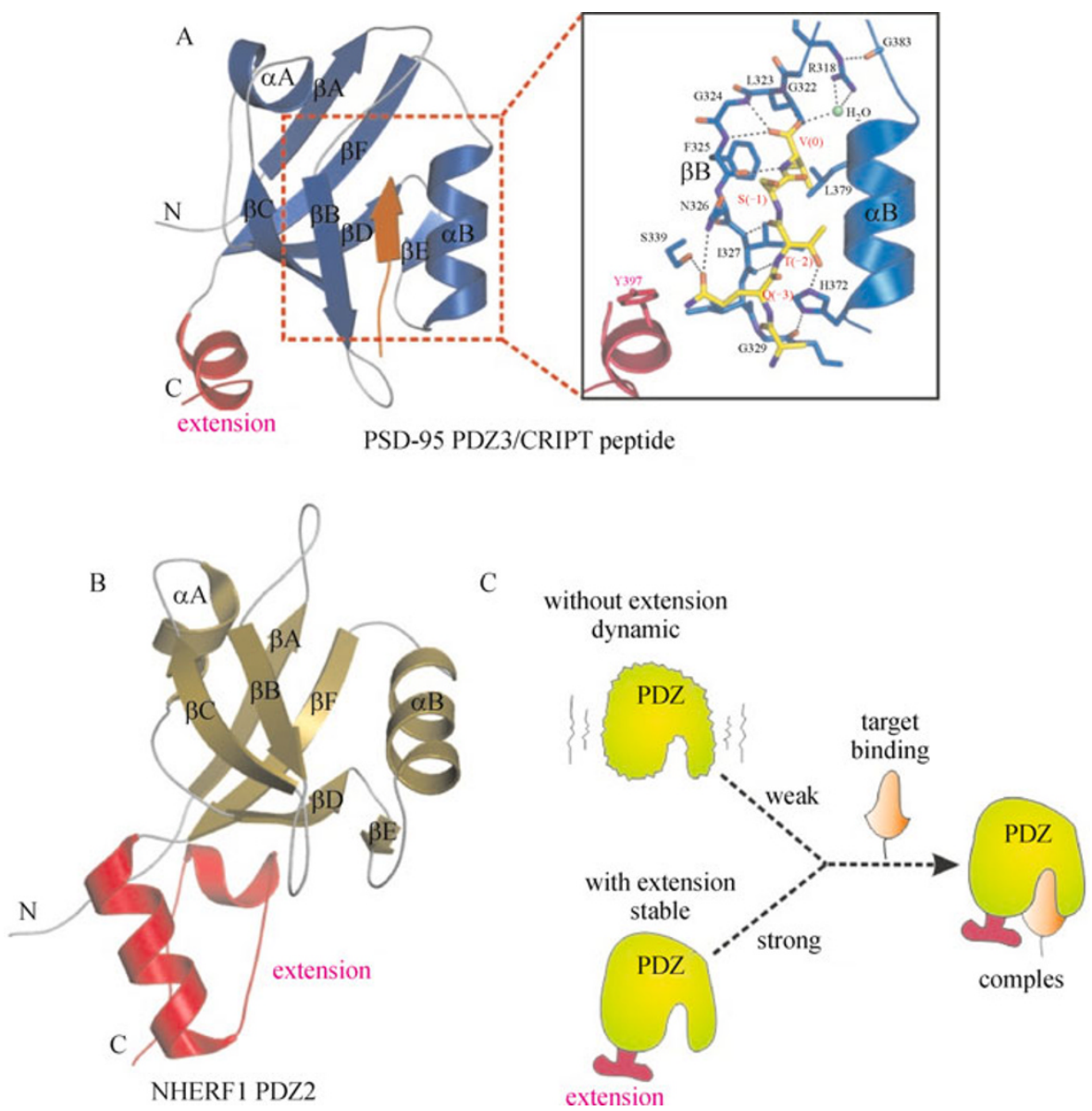

Figure 3. Protein dynamics-based modulation of target binding affinity. Panel A shows the structure of the third PDZ domain (PDZ3) of PSD-95 bound to the CRIPT peptide (orange), with the inset showing the molecular details of the interaction (PDB ID: 1BE9). Panel $B$ shows the free form of the second PDZ domain (PDZ2) of NHERF1 (PDB ID: 2KJD). Both PSD-95 PDZ3 and NHERF1 PDZ2 have an $\alpha$-helical extension (pink) at the C-terminal end. The secondary structure elements of the PDZ domains are labeled. When the extension is removed, the dynamic (or conformational) state of the main PDZ changes, leading to an effect on binding; for example, Panel $\mathrm{C}$ is a schematic diagram showing that the truncated PDZ domain is more dynamic and so binds to the target with weaker affinity compared to the extended PDZ domain, which is more stable and has stronger target binding affinity.

(GIn) of the peptide to the hydroxyl oxygen of Tyr397. Combined with the finding that the canonical fold is maintained even after removal of the additional helix, a plausible initial conclusion would be that the additional helix is of little structural or functional significance.

It is then surprising to find that removal of the helical appendage can result in a $\sim 25$-fold decrease in binding affinity for the CRIPT peptide. Isothermal titration calorimetry showed that this reduction is entirely entropic in nature-an effect which cannot be easily explained by differences in solvation because the additional helix does not form part of the ligand binding pocket (Petit et al., 2009). How then can one explain this entropy difference? NMR relaxation measurements of the protein dynamics were apparently uninformative, showing that backbone motion remains largely unaffected after truncation. However, the key result from the dynamics study is that side-chain flexibility increases significantly for the truncated domain, but can be restored to a state like that of the extended domain upon ligand binding, suggesting that affinity reduction is mainly an effect of sidechain entropy (Petit et al., 2009). Thus, for PSD-95 PDZ3, the helical extension indirectly affects binding affinity by specific modulation of side-chain dynamics. Interestingly, this mechanism has been considered to be allosteric because the additional helix is distal from the peptide ligand and because the affinity change is driven by dynamic fluctuations outside the ligand binding pocket (Petit et al., 2009).

It is tempting to speculate that the additional helix could have a defined biologic role. Because ligand binding affinity appears to require the presence of a properly packed helical extension, targeted disruption of this association between the extension and the core domain could potentially act as a 
regulatory mechanism which could be triggered through phosphorylation, for example. Indeed, it has been shown that PSD-95 can be phosphorylated at many residues, including Tyr397 located in the additional helix (Ballif et al., 2008). Because the phenol ring of Tyr397 forms part of the packing interface, phosphorylation would disrupt the packing or lead to loss of the extension structure, resulting in an allostery-mediated effect on ligand binding. It would be interesting to test whether Tyr397 phosphorylation is used to regulate PSD-95 PDZ3/target interaction in vivo.

Another example of an extension being important for ligand binding has been reported for NHERF1, which is a PDZcontaining protein involved in assembling signaling complexes and regulating the endocytic recycling of the cystic fibrosis transmembrane conductance regulator (CFTR) among other functions (Short et al., 1998; Weinman et al., 2006). With a helical extension attached after the canonical domain (as shown in Fig. 3B), the second PDZ domain (PDZ2) is considerably more stable in denaturing conditions and also able to bind a CFTR peptide with increased ( 10-fold higher) affinity. Similar to PSD-95 PDZ3, the extension does not seem to have a large impact on the fold of the canonical domain; superimposition of the structures of an extended and truncated form show that there is little deviation in the backbone conformation (Bhattacharya et al., 2010). It certainly seems that the NHERF1 PDZ2 extension and PSD-95 PDZ3 extension have many conserved structural and functional features.

Thus, as illustrated in Fig. $3 \mathrm{C}$, one potential role of PDZ extensions is to modulate the protein dynamics of the domain, which is functionally significant if the target binding affinity can be affected and biologically relevant if this mechanism can be regulated in the cellular environment. PSD-95 PDZ3 is a special case of an extension that modulates target binding affinity through specific side-chain dynamics; however, it is possible that extensions of other PDZ domains may affect backbone flexibility or conformation to modulate binding of the core PDZ to peptide ligands and/or other ligand types, such as lipids. Given that there may be a significant number of extended PDZ domains (Fig. 2), it will be worth examining whether those extensions help to regulate PDZ function in a biological setting.

\section{Provision of binding sites for multi-protein assembly}

A highly conserved protein involved in establishing metazoan cell polarity is the Partitioning-defective protein Par-6, which binds the Rho GTPase Cdc42 in a GTP-dependent manner through its CRIB (Cdc42/Rac interactive binding) motif. The CRIB motif of Par- 6 is unusual in that it lacks some of the conserved residues of a typical CRIB sequence and, importantly, cannot bind Cdc42 if the adjacent PDZ is incomplete (Joberty et al., 2000; Lin et al., 2000). The CRIB motif combined with the PDZ domain form an extended PDZ domain, which, unlike the aforementioned examples, has its extension at the $\mathrm{N}$ terminus. When overexpressed in MadinDarby Canine Kidney (MDCK) cells, this extended PDZ domain is sufficient in inhibiting the formation of tight junctions, mimicking the behavior of full-length Par-6 overexpression; however, overexpression of constructs encoding either the CRIB motif attached to an incomplete PDZ domain or the canonical PDZ domain only are unable to produce the same dominant negative effect, suggesting that the extended domain is a biologically functional unit (Joberty et al., 2000; Gao et al., 2002; Garrard et al., 2003).

The PDZ domain of Par-6 is one of the few known PDZ domains that has a binding groove capable of interacting with an internal protein segment (e.g., an $\mathrm{N}$-terminal region of Pals1, a scaffold protein associated with tight junctions); but, it is also known to bind more classically to C-terminal motifs (e.g., the $\mathrm{C}$ terminus of $\mathrm{Crb3}$, a small transmembrane protein) (Hurd et al., 2003; Lemmers et al., 2004; Penkert et al., 2004). In COS7 cells, activated forms of Cdc42 can modulate the binding of Par-6 to Pals 1 , suggesting that there may be some functional connection between the CRIB motif and the PDZ domain of Par-6 (Hurd et al., 2003). Although Cdc42 binding does not seem to affect Par- 6 binding to Pals 1 in vitro, it does induce a $\sim 13$-fold increase in binding affinity for the $C$ terminus of an artificial peptide (-VKESLV-COOH). Furthermore, unlike overexpression of the extended Par-6 PDZ domain, overexpression of a mutant extended domain that maintains the ability to bind $\mathrm{Cdc} 42$ and internal protein targets but loses its affinity for C-terminal peptides does not inhibit tight junction formation in MDCK cells, supporting the biologic importance of the coupling between Cdc42 binding and Cterminal peptide-ligand binding (Peterson et al., 2004).

What is the structural mechanism underpinning the interaction between the CRIB motif and the PDZ domain of Par-6? Similar to the case of PSD-95 PDZ3, the conformation of the core Par- 6 PDZ domain is largely unaffected by the extension, as both truncated and extended forms have almost identical NMR resonances for the PDZ domain (Garrard et al., 2003). The difference between PSD-95 PDZ3 and Par-6 PDZ is that the extension of Par-6 PDZ does not pack up against the core fold; instead, it is largely unstructured in solution (Fig. $4 \mathrm{~A}$ ) and, therefore, is unlikely to modulate the binding properties of the PDZ domain through an allosteric pathway in such a form. Strikingly, the conformation of the extension changes significantly when bound to $\mathrm{Cdc} 42$, adopting a $\beta$ sheet that is anti-parallel to both the $\beta 2$ of $C d c 42$ and the $\beta A$ of Par-6 PDZ (Fig. 4B). Combined with structural rearrangements of $\alpha A$ and its adjacent loops caused by direct contact between $\alpha A$ and $C d c 42$, the altered packing of the CRIB motif to the core PDZ may help restrict the flexibility of the $\beta A-\beta B$ loop, which is part of the binding pocket and highly dynamic in the absence of $\mathrm{Cdc} 42$, thereby reducing the entropic cost of binding. Another important structural change within the binding pocket is the altered alignment of $\mathrm{aB}$, which shifts 


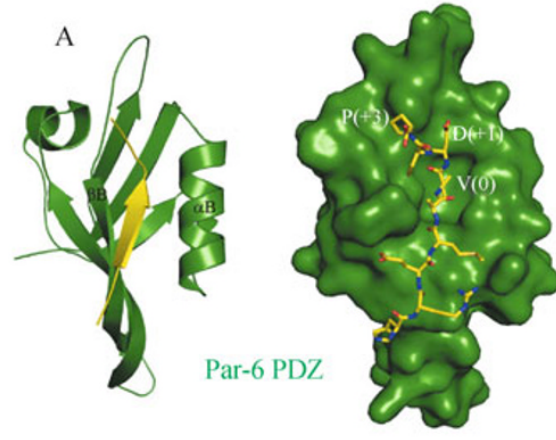

Par6 PDZ/Pals1 internal peptide

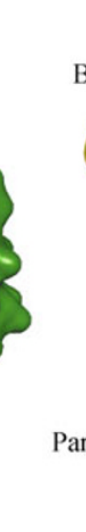

B

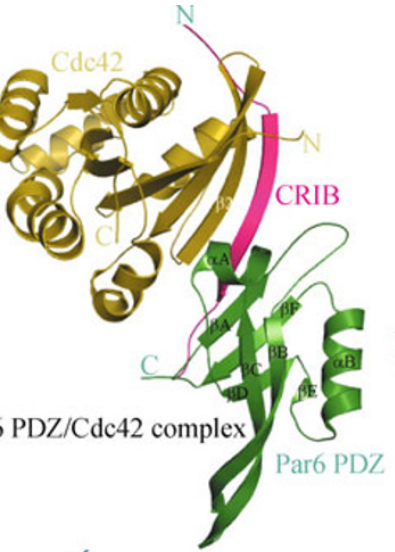

C

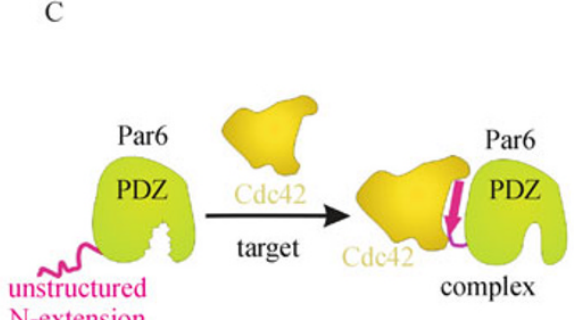

F

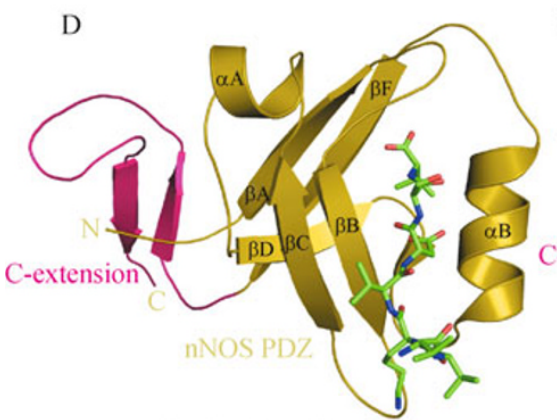

nNOS PDZ/peptide complex
E

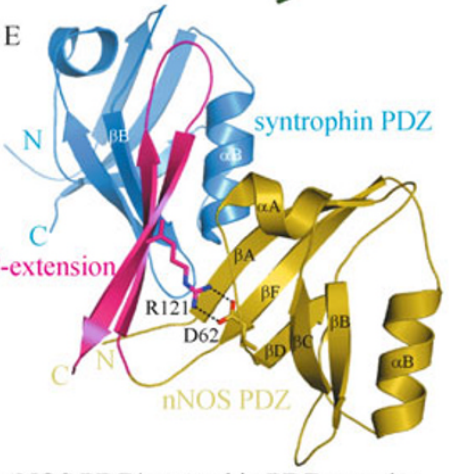

$\mathrm{N}$-extension

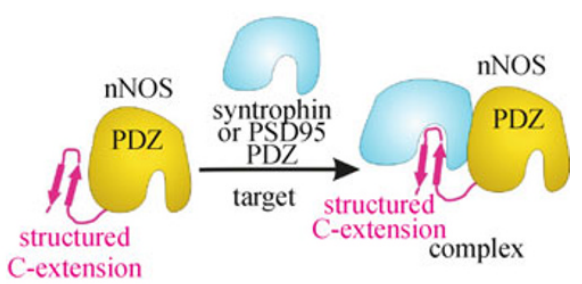

nNOS PDZ/syntrophin PDZ complex

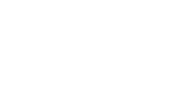

Figure 4. Provision of binding sites for macro-molecular assembly. Par-6 PDZ can bind an internal peptide region (yellow) as shown in panel A (PDB ID: 1X8S). In panel B, Par-6 PDZ has an N-terminal extension (pink) that contains a CRIB motif; the extension forms a $\beta$-sheet when bound to Cdc42 (yellow, PDB ID: 1NF3). Panel $C$ provides a cartoon representation of this interaction between Par-6 PDZ and Cdc42. Binding of Cdc42 stabilizes the target binding pocket. Panel D shows the extended nNOS PDZ domain with its ligand binding pocket occupied by a C-terminal peptide (green, PDB ID: 1B8Q). The extension of nNOS PDZ (pink) forms a semiflexible $\beta$-hairpin that becomes more structured when bound to syntrophin PDZ (blue), as shown in Panel E (PDB ID: 1QAV). Panel F is a cartoon representation of this interaction. The second PDZ domain (PDZ2) of PSD-95 can also bind the extension of nNOS PDZ.

into an orientation that is considered more favorable for peptide binding. Thus, binding of Cdc42 to the CRIB motif can result in a change in the conformation (and dynamic state) of the PDZ domain from one that has low affinity for a C-terminal peptide ligand to one that has high affinity for the same ligand. In support of this two-state model for the PDZ, the high-affinity conformation can be induced by $\mathrm{C}$-terminal peptide-ligand binding when $\mathrm{Cdc} 42$ is absent. As $\mathrm{Cdc} 42$ binds to a region of the extended PDZ distinct from the binding groove, the difference in C-terminal peptide-ligand binding affinities between the Cdc42-free and Cdc42-bound states is mediated by an allosteric transition in the PDZ domain (Peterson et al., 2004).

Although the aforementioned structural analysis explains how binding of Par-6 PDZ to C-terminal peptide ligands is regulated by $\mathrm{Cdc42}$, it still remains confusing why binding to internal peptide ligands, such as that of Pals1, is not regulated in a similar manner. The structure of Par- 6 PDZ bound to an internal peptide clarifies this apparent anomaly. When compared to the structure of Par-6 PDZ bound to a Cterminal peptide, Par-6 PDZ adopts a 'deformed' conformation when bound to a Pals1 peptide; two loops, which connect the $\beta A$ and $\beta B$ strands, and the $\beta B$ and $\beta C$ strands, have substantial deviations between the two structures, with distances up to $7 \AA$ in the $\beta A-\beta B$ loop. These structural changes decouple $\mathrm{Cdc4} 2$ binding and internal-peptide binding to the PDZ (Penkert et al., 2004). In all, these structural studies emphasize how PDZ extensions can be involved in a complex system of protein-protein interactions.

Another noteworthy example of a PDZ domain using its extension to assemble macro-molecular complexes is the PDZ of nNOS, the neuron- and muscle-specific isoform of the enzyme that produces the second messenger nitric oxide (NO) (Dawson et al., 1992; Bredt and Snyder, 1994; Huang et al., 1994). In neurons, association of nNOS with PSD-95 is thought to couple NO production to NMDA receptor activation (Brenman et al., 1995; Thomas et al., 1998). In muscle cells, association with syntrophin couples NO production to muscle 
contraction by forming the dystrophin complex; impaired functioning of this signaling pathway in Duchenne muscular dystrophy may contribute to muscle degeneration (Brenman et al., 1995).

The extended PDZ domain of nNOS, which has its extension after the canonical PDZ domain, forms a heterodimer with either the second PDZ domain (PDZ2) of PSD-95 or the single PDZ domain of a1-syntrophin (Brenman et al., 1996a, b). According to biochemical studies, formation of the heterodimer is abolished if the extended domain is truncated at either the $\mathrm{N}$ - or $\mathrm{C}$-terminus, suggesting that an intact PDZ domain and extension is required for complex assembly (Christopherson et al., 1999; Tochio et al., 2000). It probably comes as a surprise then that the extension of nNOS PDZ by itself can bind to PSD-95 PDZ2; however, this interaction was shown using NMR, which is a sensitive approach for detecting protein-protein interactions (Wang et al., 2000).

It has been shown that the binding pocket of nNOS PDZ can itself interact with C-terminal peptides (Tochio et al., 1999). In an unbound state, the extension of nNOS PDZ does not affect the peptide binding affinity of the core PDZ, as both the extended and canonical forms have similar affinities for the C-terminal peptide (Tochio et al., 1999). The extended domain is more stable than the canonical domain in denaturing conditions (Christopherson et al., 1999); however, the stability of the fold does not seem to influence the binding affinity of the PDZ in this case. It is unknown whether a bound extension can modulate the binding properties of the core nNOS PDZ in a manner similar to that of Par-6 PDZ as discussed above.

A few unique points need to be mentioned from structural studies of the nNOS PDZ. Like all of the extended PDZ domains discussed so far, the presence of the extension for nNOS PDZ has almost no effect on the fold of the main PDZ domain, as both canonical and extended forms have similar NMR resonances for the main PDZ. Whereas the extension of Par-6 PDZ is largely unstructured when its binding partner is absent, the extension of nNOS PDZ forms a semi-flexible $\beta$ hairpin that packs loosely against the core PDZ in its unbound state (Tochio et al., 2000) (Fig. 4D) and can exist, albeit transiently, in a native-like $\beta$-hairpin conformation by itself in solution (Wang et al., 2000). The extension is pinned against the main PDZ domain through a buried salt bridge between Arg121 in the second $\beta$-strand of the $\beta$-finger extension and Asp62 in the $\beta D$ strand of the PDZ domain. Mutation of Arg121 to Gln has no affect on the fold of the PDZ domain but destabilizes the extension and results in an interaction with PSD-95 PDZ2 that is barely detectable by SPR, suggesting that a structured extension is required for nNOS heterodimerization (Tochio et al., 2000). In fact, the extension is the main contact region for both PSD-95 PDZ2 and a1-syntrophin PDZ, with additional contacts made at $\beta A$ of nNOS PDZ (Hillier et al., 1999). Upon formation of a hetero-dimer, the extension becomes stabilized but the fold of the core PDZ is largely unaffected (Fig. 4E) (Tochio et al., 2000), suggesting that if a coupling between the bound extension and the binding affinity of the PDZ exists, it is not as obvious as that of the extended Par6 PDZ. Nonetheless, these studies support the view that the extended nNOS PDZ domain is an important structural entity.

We have so far provided two examples of PDZ extensions being used for macro-molecular assembly. In both cases, binding of a protein partner to the extension structurally stabilizes the extension as illustrated in panels $\mathrm{C}$ and $\mathrm{F}$ of Fig. 4. The extended Par-6 PDZ is particularly interesting because binding of $\mathrm{Cdc} 42$ to its extension initiates an allosteric transition of the main PDZ into a conformation that has higher affinity for a C-terminal peptide ligand. In the bound form, the PDZ extension adopts Role 1 as well, but the conformational shift in the Par-6 PDZ is also a result of contacts between Cdc42 and the PDZ. Nonetheless, it is possible for PDZ extensions to adopt multiple roles, customizing the function of the PDZ to suit the dynamic and complex environment within the cell.

\section{Structural integration of multi-domain modules}

Association of a glutamate receptor-interacting protein called GRIP1 with the GluR2 subunit of the AMPA receptor is critically important for synaptic targeting and clustering of the receptor (Dong et al., 1999; Osten et al., 2000). The Cterminal tail of GluR2 binds to the fourth and fifth PDZ domain (PDZ4-PDZ5) module of GRIP1; but, surprisingly, cannot bind the isolated PDZ4 and PDZ5 domains (Zhang et al., 2001). This is because PDZ4 contains a distorted binding pocket that is unfavorable for peptide-ligand binding; and because PDZ5 is unstructured in solution, regaining the canonical PDZ fold when it is in the PDZ4-PDZ5 tandem. The structure of this tandem, which is shown in Fig. $5 \mathrm{~A}$, highlights two important PDZ extensions: 1) an unstructured extension $\mathrm{N}$-terminal of PDZ4, and 2) an extension, which can be classified as belonging to either PDZ4 or PDZ5, that acts as a linker joining the two PDZ domains (Feng et al., 2003). The sequence of this 'linking' extension is highly conserved across GRIP proteins, suggesting that it may have an important structural or functional role. In support of this, proper folding and function of the PDZ4-PDZ5 module requires the extension to be intact (Zhang et al., 2001). In the tandem structure, the second half of the extension that links the two PDZ domains adopts a $\beta$-sheet that packs up against the $\beta A$ of the folded PDZ5, further supporting the existence of a structural role for this extension. The tandem structure also suggests that the N-terminal PDZ4 extension may be important because it interacts with the central PDZ extension. Indeed, the Nterminal PDZ4 extension is required for the interaction of PDZ4-PDZ5 with GluR2 and also increases the stability of the PDZ4-PDZ5 module in urea (Feng et al., 2003). Thus, the GRIP1 PDZ4-PDZ5 module contains at least two PDZ 
A
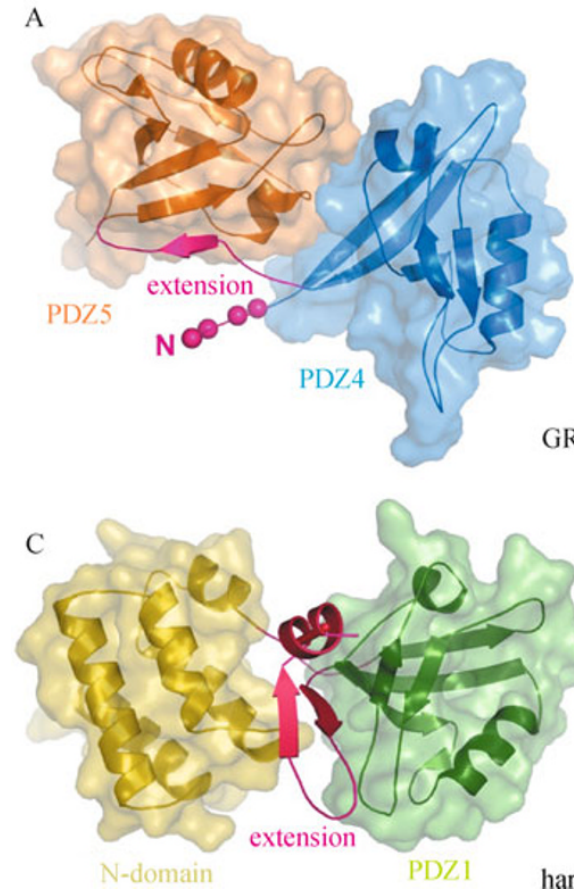

B

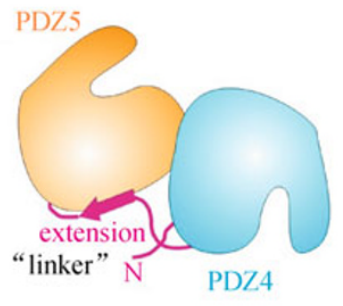

GRIP PDZ45 tandem

\section{D}

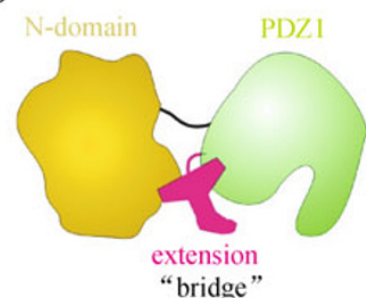

harmonin NPDZ1

Figure 5. Structural integration of multi-domain modules. The fourth and fifth PDZ domains of GRIP form the PDZ4-PDZ5 tandem as shown in Panel A (PDB ID: 1P1D). The structure and function of this tandem requires an extension (pink) that connects the two domains (Panel B). The structural arrangement of the N-domain and the first PDZ domain (PDZ1) of harmonin (PDB 3K1R) also requires an extension (pink) that forms a $\beta$-hairpin and an $\alpha$-helix and packs up against both domains (Panel $C$ and $D$ ).

extensions that seem to have structural and functional roles.

Whereas PDZ extensions can be used to structurally organize two PDZ domains, as described for GRIP1 PDZ4PDZ5 above, PDZ domains can also be coupled to other types of domains, as is the case for an N-terminal region of harmonin. Harmonin is a scaffold protein that organizes a network of protein-protein interactions implicated in Usher syndrome, the most common form of hereditary deafblindness in humans (El-Amraoui and Petit, 2005). One key interaction required for the proper development of cilia in photoreceptor and hair cells is between harmonin and Sans (El-Amraoui and Petit, 2005). It has been shown that the binding of the $\mathrm{C}$-terminal PBM of Sans to the first PDZ domain (PDZ1) of harmonin requires PDZ1 to be embedded in the $\mathrm{N}$ PDZ1 supramodule, which comprises the $\mathrm{N}$-terminal domain, PDZ1, and the PDZ1 extension. This is because PDZ1 by itself appears to be misfolded, and can only be bacterially expressed in a soluble form when it is attached to its preceding N-terminal domain; and because removal of the PDZ1 extension results in a truncated N-PDZ1 module that can no longer bind the Sans PBM. The structure of the $\mathrm{N}$ PDZ1 module, which is depicted in Fig. 5C, reveals the critical role that the PDZ1 extension has in integrating the $\mathrm{N}$-terminal domain with PDZ1. The PDZ1 extension, which is composed of a $\beta$-hairpin followed by an $\alpha$-helix, wraps around and makes extensive contacts with both the $\mathrm{N}$-terminal and PDZ1 domains. More specifically, the interface between the $\mathrm{N}$ domain and the PDZ1 extension is stabilized by many hydrophobic and electrostatic interactions, burying a substantial surface area of $423 \AA^{2}$, whereas the interface between PDZ1 and its extension is primarily mediated by hydrophobic interactions. The amino acid residues forming the two interfaces are evolutionarily conserved, supporting the importance of these residues, and therefore the PDZ1 extension, for the assembly of the N-PDZ1 module (Yan et al., 2010)

Both GRIP1 and harmonin use PDZ extensions to organize the spatial configuration of their domains (Fig. 5B and 5D). The extension may lie between two domains of interest or may sit on the periphery of a multi-domain module. Although we have focused on extensions that have relatively short sequences in this section, it is evident that the structure and function of the extended PDZ within the module also relies heavily on its neighboring domain-we discuss PDZ extensions that are entire domains themselves below.

\section{Expansion of the target ligand binding pocket}

So far, we have proposed roles of PDZ extensions that indirectly affect the binding properties of the main PDZ domain; is it possible that PDZ extensions can directly affect 
PDZ function? We refer again to the N-PDZ1 module of harmonin to show that this is possible. As mentioned above, the N-PDZ1 module binds to the C-terminal PBM of Sans; in fact, the N-PDZ1 module binds with higher affinity to the Sans SAM-PBM, a longer C-terminal region of Sans that has a SAM domain in addition to the PBM, to form a tightly bound complex (Yan et al., 2010). Although the structure of the complex (Fig. 6A) shows a highly unusual PDZ-mediated protein-protein interaction between harmonin PDZ1 and Sans SAM, here we are specifically interested in the last eight residues of Sans that immediately follows the SAM domain. Initial analysis of the structure of the complex, reveals that the last three residues of Sans binds to harmonin PDZ1 via a classical type I PDZ domain/ligand mode, with T459 and L461 of Sans making typical contacts with residues of PDZ1. What is not typical is that residues of Sans upstream of the classical four-residue PBM, i.e., A455 and L456, form direct contacts with the harmonin PDZ1 extension (Fig. 6B and 6C) (Yan et al., 2010). As the harmonin PDZ1 extension is directly involved in binding to the ligand, the extension is effectively expanding the binding pocket of PDZ1.

Thus, in this role, the PDZ extension directly contacts the bound ligand, and therefore, has a direct effect on ligand binding affinity and specificity. As PDZ domains can often bind multiple ligands with comparable affinities, mechanisms that control their specificity are important for understanding their function in the cell, where a mixture of potential protein partners is present. With an expanded target ligand binding pocket, the PDZ binds to a region of the target that has a length longer than the canonical four amino acid residues. Other PDZ domains have been shown to bind atypically long target sequences (Stiffler et al., 2007; Tonikian et al., 2008). It will be interesting to investigate whether extensions contribute to the target binding specificity for other PDZ domains.

\section{FUTURE PERSPECTIVES}

Our analysis of the roles of PDZ extensions combined with our newly-developed database of predictions provides a foundation for further study on PDZ extensions. Helical extensions predicted to be at the C-terminal end of many PDZ domains, such as some PDZ domains from NHERF proteins and the first PDZ domain of Partitioning-defective protein Par-3 (Fig. 7A), may modulate the dynamics of the main PDZ domain. Further structural and biochemical studies would be needed to verify the presence and role of the predicted extension.

One PDZ extension that has convincing biochemical (but no structural) data suggesting that it has an important role in integrating multiple domains is the one that links the PDZ and
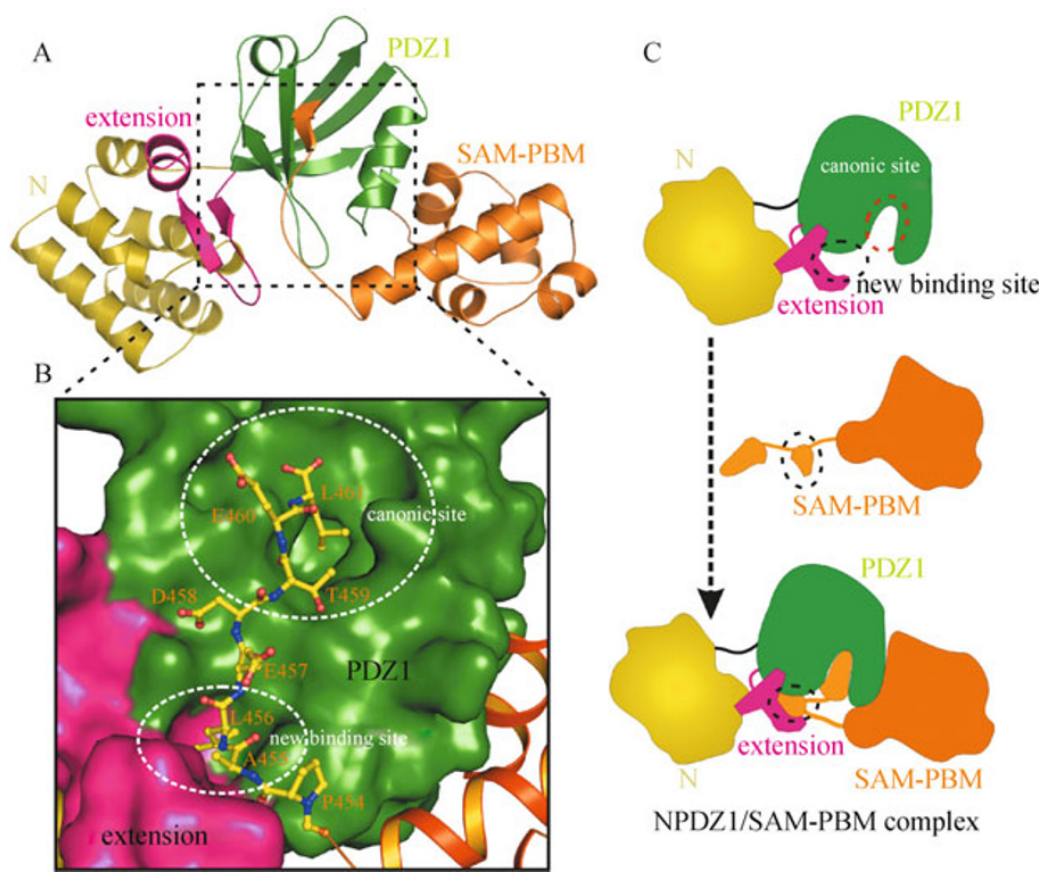

Figure 6. Expansion of the target ligand binding pocket. The N-terminal and first PDZ domain (PDZ1) of harmonin form the NPDZ1 module that binds to a C-terminal region of SAM (which includes the PDZ binding motif, PBM) through PDZ1 (Panel A, PDB ID: 3K1R). In Panel B, a detailed molecular description of this interaction is shown and the residues of the ligand are labeled. Panel $B$ highlights the canonical and the new binding site, which involves the extension (pink) and extends the canonical target binding groove. The residues involved in this new binding site are circled. Panel $\mathrm{C}$ shows a cartoon representation of the complex formation between harmonin N-PDZ1 and SAM-PBM, which involves the PDZ extension. 
A

helical extensions

Par3

-NTD \begin{tabular}{ll:l} 
PDZ & PDZ & PDZ \\
\hdashline
\end{tabular}

DLG1

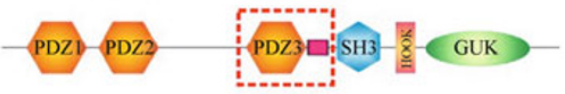

B

homo-supramodules

Prex 1

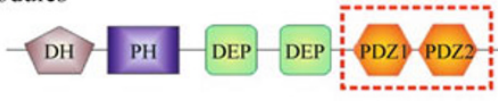

Scribble

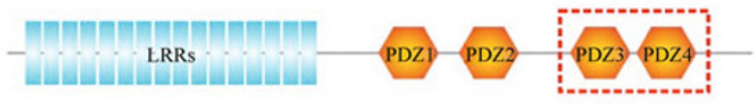

DLG5

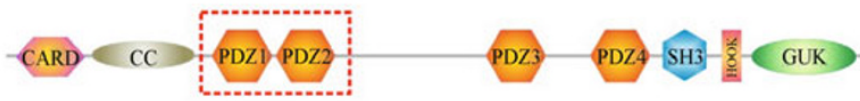

C

hetero-supramodules

Tiam 1/2
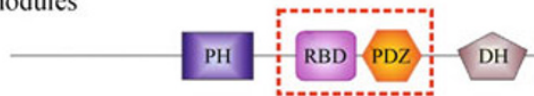

PH

MAGI1/2/3

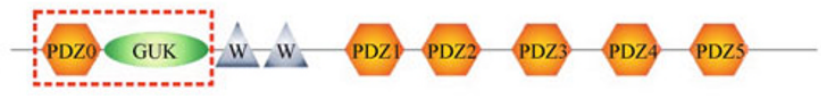

domain keys

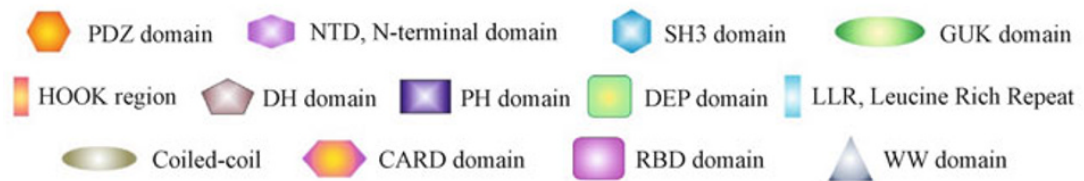

Figure 7. PDZ extensions and supramodules. This figure shows the domain architecture of selected PDZ-containing proteins that might have interesting extended PDZ domains. Panel A shows two proteins that might have a-helical extensions (red box). Of course, extensions can be domains themselves. When two or more domains are structurally or functionally coupled, they are termed supramodules. Some possible supramodules are highlighted in Panel B and C. When a supramodule consists only of one type of domain, it is called a homo-supramodule; when a supramodule consists of more than one type of domain, it is called a heterosupramodule. A key describing the different types of domains within these proteins are shown at the bottom of the panel.

SH3 domains within the PDZ-SH3-GUK module of the Drosophila tumor suppressor Discs Large 1 (Dlg1; see Fig. 7A). Dlg1 facilitates the organization of signal transduction pathways in a number of polarized cells, and is a well-studied and evolutionarily-conserved member of the MAGUK protein family. Dlg1 interacts with the synaptic protein GukHolder (GukH) to carry out such functions as the localization of the tumor suppressor Scribble. Biochemical experiments have shown that GukH binds to a composite site formed by the $\mathrm{SH} 3$ and GUK domains of the PDZ-SH3-GUK module of Dlg1. Importantly, this interaction can be abolished by removal of the PDZ domain from the PDZ-SH3-GUK module or reduced significantly by binding of the C-terminal peptide of CRIPT to the PDZ domain. This coupling between the PDZ domain and the SH3-GUK module seems to require the PDZ extension, which adopts a helical structure that packs up against the core PDZ. Deletion of either the first or second half of the extension or replacement of the extension with an unstructured sequence (composed of only Gly and Ser) results in a PDZ-SH3-GUK mutant that cannot bind GukH, suggesting that both the length and conformation of the PDZ extension is required to couple the PDZ domain to the SH3-GUK domains (Qian and Prehoda, 2006). Detailed structural information will be required to explain how the PDZ extension between PDZ and the SH3-GUK module helps to couple two separate binding events: peptide-ligand binding to the PDZ and GukH binding to the SH3-GUK module.

\section{Domains as extensions and supramodules}

We have thus far focused on extensions that are short regions immediately preceding and following the canonical PDZ domain, showing that these extensions can have a structural and/or functional effect on the core PDZ domain. However, 
there is no reason why extensions cannot be entire domains themselves. Both PDZ1 of harmonin and PDZ5 of GRIP1 require a neighboring domain to be tightly packed up against for proper structure and function, suggesting that domains adjacent to a PDZ domain can adopt the roles of PDZ extensions that were proposed above. We predict that there exist PDZ domains that are extended by entire domains (either with or without additional short extensions), such as the PDZO-GUK module of membrane associated guanylate kinase, WW and PDZ domain containing 1 (MAGI1). A region of a protein containing two or more domains which are structurally and functionally coupled has been referred to as a supramodule, a feature that is increasingly being recognized in many proteins (Feng and Zhang, 2009). Figure 7B and 7C highlight some PDZ-containing proteins that may harbour supramodules.

In a way, supramodules can be viewed as a specific type of multi-domain complex that is distinguished by having all constituent domains of the complex within one protein. Of course, multi-domain complexes can also be formed by the association of domains from different proteins or from different copies of the same protein. An oligomer is another specific type of a multi-domain complex. Although domains within these complexes do not strictly extend the sequence of a PDZ domain that participates in the complex, they often affect (and thus extend) the structure and function the PDZ domain. For example, the second PDZ domain (PDZ2) of $Z O-1$, a protein with critical functions in formation and maintenance of intercellular junctions, forms a domain-swapped dimer; the two PDZ2 molecules become interlocked, and each PDZ2 has an extended conformation compared to the canonical fold. The structure of this dimer bound to two Cterminal peptides of connexin 43 (Cx43), a protein involved in gap junction formation and regulation, has been solved (Chen et al., 2008). The charge-charge interaction network formed by residues in the PDZ dimer interface and upstream residues of the Cx43 peptide not only increases the specificity of each PDZ for the CX43 peptide but may also act as a site for phosphorylation-mediated regulation. In the dimer, if one PDZ domain was viewed as the extension of the other, then the PDZ domain acting as the extension would be adopting Role 4 that was proposed above. The outcome of the domain swapping is to create a second binding site (i.e., an extended target binding pocket) at the interface of the swapped PDZ dimer. It is interesting to note that formation of the domainswapped dimer requires that $\beta B$ and $\beta C$ form a continuous $\beta$ sheet; in other words, the loop that usually sits between $\beta B$ and $\beta C$ is replaced by an additional $\beta$-sheet that extends $\beta B$ or $\beta C$. This is a unique example of an extension that is hidden within the domain, rather than being positioned outside the canonical domain boundary. It was noted that such domain swapped PDZ dimerization may occur in other PDZ domain proteins (Chen et al., 2008). In view of the large number of PDZ domains in mammalian genomes, it is highly likely that there are still other forms of PDZ domain extensions that remain to be discovered in the future.

\section{CONCLUSION}

We initially defined domain extensions as structured regions that lie immediately outside the canonical domain boundary, on either the $\mathrm{N}$ - or $\mathrm{C}$-terminal side, and then applied this definition to search for extended PDZ domains using bioinformatics. Our results, which can be easily accessed from our newly-developed database/website (http://bcz102. ust.hk/pdzex/), suggest that extended PDZ domains are not uncommon. Indeed, the PDZ domains that are discussed above have extensions that adopt $\alpha$-helical and/or $\beta$-sheet conformations. Through the course of this review, we have also shown that some PDZ extensions are unstructured-the $\mathrm{N}$-terminal extension of Par-6 PDZ becomes structured when bound to Cdc42; and the N-terminal extension of GRIP1 PDZ4 is important for structure, stability, and function of the PDZ4-PDZ5 module. Thus, both structured and disordered extensions may affect the structure and function of the core PDZ domain. We have also considered extensions that are entire domains. It is therefore likely that extended PDZ domains are more prevalent than we expect. Consistent with our analysis, recent systematic PDZ domain/target interaction analysis revealed that over $30 \%$ of PDZ domains could not be obtained in bacterial overexpression systems (Stiffler et al., 2007; Wu et al., 2007; Tonikian et al., 2008). Based on our own experience in working with a large number of PDZ domains in the past decade, it is important to evaluate the boundaries of PDZ domains with great care as many PDZ domains require extended sequences for proper folding.

We have identified at least four roles of PDZ extensions, which act as a starting point for understanding domain extensions in general. These roles are: 1) protein dynamicsbased modulation of target binding affinity, 2) provision of binding sites for macro-molecular assembly, 3) structural integration of multi-domain modules, and 4) expansion of the target ligand binding pocket. These four roles are not mutually exclusive; the extension of harmonin PDZ1, for example, adopts at least roles 3 and 4 . The PDZ protein examples that we have discussed here are conserved from fly to human, indicating that the extended PDZ domain is a common property throughout evolution.

In this review, we have focused on the functional and structural effects of PDZ extensions. It is possible that these effects can be regulated in a biologic setting through events such as phosphorylation. The extension of PSD-95 PDZ3, for example, contains a verified phosphorylation site. We have not discussed in detail the effect of PDZ extensions on folding, but we note here that there is one study which showed that when PSD-95 PDZ3 does not have its extension, it has a higher tendency to be trapped as a folding intermediate (Feng et al., 2005). The PDZ extension also has a role in protein 
stability, for both the nNOS PDZ domain and the GRIP PDZ4PDZ5 module, where their stability in denaturing conditions is increased when their PDZ extensions are present. In this review, we have highlighted the importance of PDZ extensions, and we propose that other domains may also have extensions that have important roles-it sometimes pays to look outside the canonical boundary. It should be noticed that extension sequences outside canonical protein functional domains are a well known concept. The most common example in using extension sequences to modulate protein core functions is perhaps enzymes, as enzymes often build in regulatory mechanisms using such extension sequences. We should be aware that extension sequence-mediated structure and functional modulation is also a quite common property for modular protein-protein interaction domains such as PDZ domains discussed here.

\section{ACKNOWLEDGEMENTS}

The work in MZ's laboratory was supported by grants from the Research Grants Council of Hong Kong to M.Z. (HKUST663407, 663808, 664009, CA07/08.SC01, SEG_HKUST06 and AoE/B-15/01II). The NMR spectrometer used in this work was purchased with funds donated to BRI by the Hong Kong Jockey Club. CKW is supported by a fellowship from the NHMRC of Australia (536578).

\section{REFERENCES}

Ballif, B.A., Carey, G.R., Sunyaev, S.R., and Gygi, S.P. (2008). Largescale identification and evolution indexing of tyrosine phosphorylation sites from murine brain. J Proteome Res 7, 311-318.

Bateman, A., Coin, L., Durbin, R., Finn, R.D., Hollich, V., GriffithsJones, S., Khanna, A., Marshall, M., Moxon, S., Sonnhammer, E. L., et al. (2004). The Pfam protein families database. Nucleic Acids Res 32, D138-D141.

Bhattacharya, S., Dai, Z., Li, J., Baxter, S., Callaway, D.J., Cowburn, D., and Bu, Z. (2010). A conformational switch in the scaffolding protein NHERF1 controls autoinhibition and complex formation. $J$ Biol Chem 285, 9981-9994.

Blazer, L.L., and Neubig, R.R. (2009). Small molecule protein-protein interaction inhibitors as CNS therapeutic agents: current progress and future hurdles. Neuropsychopharmacology 34, 126-141.

Bredt, D.S., and Snyder, S.H. (1994). Nitric oxide: a physiologic messenger molecule. Annu Rev Biochem 63, 175-195.

Brenman, J.E., Chao, D.S., Xia, H., Aldape, K., and Bredt, D.S. (1995). Nitric oxide synthase complexed with dystrophin and absent from skeletal muscle sarcolemma in Duchenne muscular dystrophy. Cell 82, 743-752.

Brenman, J.E., Chao, D.S., Gee, S.H., McGee, A.W., Craven, S.E., Santillano, D.R., Wu, Z., Huang, F., Xia, H., Peters, M.F., et al. (1996a). Interaction of nitric oxide synthase with the postsynaptic density protein PSD-95 and alpha1-syntrophin mediated by PDZ domains. Cell 84, 757-767.

Brenman, J.E., Christopherson, K.S., Craven, S.E., McGee, A.W., and Bredt, D.S. (1996b). Cloning and characterization of postsynaptic density 93 , a nitric oxide synthase interacting protein. $J$ Neurosci 16, 7407-7415.
Chen, J., Pan, L., Wei, Z., Zhao, Y., and Zhang, M. (2008). Domainswapped dimerization of ZO-1 PDZ2 generates specific and regulatory connexin43-binding sites. EMBO J 27, 2113-2123.

Chi, C.N., EngstrÖm, A., Gianni, S., Larsson, M., and Jemth, P. (2006). Two conserved residues govern the salt and $\mathrm{pH}$ dependencies of the binding reaction of a PDZ domain. $J$ Biol Chem 281, 36811-36818.

Christopherson, K.S., Hillier, B.J., Lim, W.A., and Bredt, D.S. (1999). PSD-95 assembles a ternary complex with the N-methyl-Daspartic acid receptor and a bivalent neuronal NO synthase PDZ domain. J Biol Chem 274, 27467-27473.

Chung, H.J., Xia, J., Scannevin, R.H., Zhang, X., and Huganir, R.L. (2000). Phosphorylation of the AMPA receptor subunit GluR2 differentially regulates its interaction with PDZ domain-containing proteins. J Neurosci 20, 7258-7267.

Cohen, A.R., Woods, D.F., Marfatia, S.M., Walther, Z., Chishti, A.H., Anderson, J.M., and Wood, D.F. (1998). Human CASK/LIN-2 binds syndecan-2 and protein 4.1 and localizes to the basolateral membrane of epithelial cells. J Cell Biol 142, 129-138.

Dawson, T.M., Dawson, V.L., and Snyder, S.H. (1992). A novel neuronal messenger molecule in brain: the free radical, nitric oxide. Ann Neurol 32, 297-311.

Dong, H., Zhang, P., Song, I., Petralia, R.S., Liao, D., and Huganir, R. L. (1999). Characterization of the glutamate receptor-interacting proteins GRIP1 and GRIP2. J Neurosci 19, 6930-6941.

Doyle, D.A., Lee, A., Lewis, J., Kim, E., Sheng, M., and MacKinnon, R. (1996). Crystal structures of a complexed and peptide-free membrane protein-binding domain: molecular basis of peptide recognition by PDZ. Cell 85, 1067-1076.

El-Amraoui, A., and Petit, C. (2005). Usher I syndrome: unravelling the mechanisms that underlie the cohesion of the growing hair bundle in inner ear sensory cells. J Cell Sci 118, 4593-4603.

Feng, W., and Zhang, M. (2009). Organization and dynamics of PDZdomain-related supramodules in the postsynaptic density. Nat Rev Neurosci 10, 87-99.

Feng, W., Shi, Y., Li, M., and Zhang, M. (2003). Tandem PDZ repeats in glutamate receptor-interacting proteins have a novel mode of PDZ domain-mediated target binding. Nat Struct Biol 10, 972-978.

Feng, H., Vu, N.D., and Bai, Y. (2005). Detection of a hidden folding intermediate of the third domain of PDZ. J Mol Biol 346, 345-353.

Frishman, D., and Argos, P. (1996). Incorporation of non-local interactions in protein secondary structure prediction from the amino acid sequence. Protein Eng 9, 133-142.

Gao, L., Joberty, G., and Macara, I.G. (2002). Assembly of epithelial tight junctions is negatively regulated by Par6. Curr Biol 12, 221-225.

Garrard, S.M., Capaldo, C.T., Gao, L., Rosen, M.K., Macara, I.G., and Tomchick, D.R. (2003). Structure of Cdc42 in a complex with the GTPase-binding domain of the cell polarity protein, Par6. EMBO J 22, 1125-1133.

Harris, B.Z., and Lim, W.A. (2001). Mechanism and role of PDZ domains in signaling complex assembly. J Cell Sci 114, 3219-3231.

Hegedüs, T., Sessler, T., Scott, R., Thelin, W., Bakos, E., Váradi, A., Szabó, K., Homolya, L., Milgram, S.L., and Sarkadi, B. (2003). Cterminal phosphorylation of MRP2 modulates its interaction with PDZ proteins. Biochem Biophys Res Commun 302, 454- 461.

Hillier, B.J., Christopherson, K.S., Prehoda, K.E., Bredt, D.S., and 
Lim, W.A. (1999). Unexpected modes of PDZ domain scaffolding revealed by structure of nNOS-syntrophin complex. Science 284 , 812-815.

Huang, Z., Huang, P.L., Panahian, N., Dalkara, T., Fishman, M.C., and Moskowitz, M.A. (1994). Effects of cerebral ischemia in mice deficient in neuronal nitric oxide synthase. Science 265, 1883-1885.

Hurd, T.W., Gao, L., Roh, M.H., Macara, I.G., and Margolis, B. (2003). Direct interaction of two polarity complexes implicated in epithelial tight junction assembly. Nat Cell Biol 5, 137-142.

Joberty, G., Petersen, C., Gao, L., and Macara, I.G. (2000). The cellpolarity protein Par6 links Par3 and atypical protein kinase $C$ to Cdc42. Nat Cell Biol 2, 531-539.

Jones, D.T. (1999). Protein secondary structure prediction based on position-specific scoring matrices. J Mol Biol 292, 195-202.

Kim, E., and Sheng, M. (2004). PDZ domain proteins of synapses. Nat Rev Neurosci 5, 771-781.

Kim, E., Niethammer, M., Rothschild, A., Jan, Y.N., and Sheng, M. (1995). Clustering of Shaker-type $K+$ channels by interaction with a family of membrane-associated guanylate kinases. Nature 378 , 85-88.

Kornau, H.C., Schenker, L.T., Kennedy, M.B., and Seeburg, P.H. (1995). Domain interaction between NMDA receptor subunits and the postsynaptic density protein PSD-95. Science 269, 1737-1740.

Lemmers, C., Michel, D., Lane-Guermonprez, L., Delgrossi, M.H., Médina, E., Arsanto, J.P., and Le Bivic, A. (2004). CRB3 binds directly to Par6 and regulates the morphogenesis of the tight junctions in mammalian epithelial cells. Mol Biol Cell 15, 1324-1333.

Lin, D., Edwards, A.S., Fawcett, J.P., Mbamalu, G., Scott, J.D., and Pawson, T. (2000). A mammalian PAR-3-PAR-6 complex implicated in Cdc42/Rac1 and aPKC signalling and cell polarity. Nat Cell Biol 2, 540-547.

Linding, R., Jensen, L.J., Diella, F., Bork, P., Gibson, T.J., and Russell, R.B. (2003). Protein disorder prediction: implications for structural proteomics. Structure 11, 1453-1459.

Lockless, S.W., and Ranganathan, R. (1999). Evolutionarily conserved pathways of energetic connectivity in protein families. Science 286, 295-299.

Mishra, P., Socolich, M., Wall, M.A., Graves, J., Wang, Z, and Ranganathan, R. (2007). Dynamic scaffolding in a $G$ proteincoupled signaling system. Cell 131, 80-92.

Mortier, E., Wuytens, G., Leenaerts, I., Hannes, F., Heung, M.Y., Degeest, G., David, G., and Zimmermann, P. (2005). Nuclear speckles and nucleoli targeting by PIP2-PDZ domain interactions. EMBO J 24, 2556-2565.

Niethammer, M., Valtschanoff, J.G., Kapoor, T.M., Allison, D.W., Weinberg, R.J., Craig, A.M., and Sheng, M. (1998). CRIPT, a novel postsynaptic protein that binds to the third PDZ domain of PSD-95/ SAP90. Neuron 20, 693-707.

Osten, P., Khatri, L., Perez, J.L., Köhr, G., Giese, G., Daly, C., Schulz, T.W., Wensky, A., Lee, L.M., and Ziff, E.B. (2000). Mutagenesis reveals a role for ABP/GRIP binding to GluR2 in synaptic surface accumulation of the AMPA receptor. Neuron 27, 313-325.

Pawson, T., and Nash, P. (2003). Assembly of cell regulatory systems through protein interaction domains. Science 300, 445-452.

Penkert, R.R., DiVittorio, H.M., and Prehoda, K.E. (2004). Internal recognition through PDZ domain plasticity in the Par-6-Pals1 complex. Nat Struct Mol Biol 11, 1122-1127.

Peterson, F.C., Penkert, R.R., Volkman, B.F., and Prehoda, K.E. (2004). Cdc42 regulates the Par-6 PDZ domain through an allosteric CRIB-PDZ transition. Mol Cell 13, 665-676.

Petit, C.M., Zhang, J., Sapienza, P.J., Fuentes, E.J., and Lee, A.L. (2009). Hidden dynamic allostery in a PDZ domain. Proc Natl Acad Sci U S A 106, 18249-18254.

Qian, Y., and Prehoda, K.E. (2006). Interdomain interactions in the tumor suppressor discs large regulate binding to the synaptic protein GukHolder. J Biol Chem 281, 35757-35763.

Rost, B., Yachdav, G., and Liu, J. (2004). The PredictProtein server. Nucleic Acids Res 32, W321-W326.

Schultz, J., Milpetz, F., Bork, P., and Ponting, C.P. (1998). SMART, a simple modular architecture research tool: identification of signaling domains. Proc Natl Acad Sci U S A 95, 5857-5864.

Short, D.B., Trotter, K.W., Reczek, D., Kreda, S.M., Bretscher, A., Boucher, R.C., Stutts, M.J., and Milgram, S.L. (1998). An apical PDZ protein anchors the cystic fibrosis transmembrane conductance regulator to the cytoskeleton. J Biol Chem 273, 19797-19801.

Stiffler, M.A., Chen, J.R., Grantcharova, V.P., Lei, Y., Fuchs, D., Allen, J.E., Zaslavskaia, L.A., and MacBeath, G. (2007). PDZ domain binding selectivity is optimized across the mouse proteome. Science 317, 364-369.

Thomas, G.D., Sander, M., Lau, K.S., Huang, P.L., Stull, J.T., and Victor, R.G. (1998). Impaired metabolic modulation of alphaadrenergic vasoconstriction in dystrophin-deficient skeletal muscle. Proc Natl Acad Sci U S A 95, 15090-15095.

Tochio, H., Zhang, Q., Mandal, P., Li, M., and Zhang, M. (1999). Solution structure of the extended neuronal nitric oxide synthase PDZ domain complexed with an associated peptide. Nat Struct Biol 6, 417-421.

Tochio, H., Mok, Y.K., Zhang, Q., Kan, H.M., Bredt, D.S., and Zhang, M. (2000). Formation of nNOS/PSD-95 PDZ dimer requires a preformed beta-finger structure from the nNOS PDZ domain. J Mol Biol 303, 359-370.

Tonikian, R., Zhang, Y., Sazinsky, S.L., Currell, B., Yeh, J.H., Reva, B., Held, H.A., Appleton, B.A., Evangelista, M., Wu, Y., et al. (2008). A specificity map for the PDZ domain family. PLoS Biol 6, e239.

Wang, P., Zhang, Q., Tochio, H., Fan, J.S., and Zhang, M. (2000). Formation of a native-like beta-hairpin finger structure of a peptide from the extended PDZ domain of neuronal nitric oxide synthase in aqueous solution. Eur J Biochem 267, 3116-3122.

Ward, J.J., McGuffin, L.J., Bryson, K., Buxton, B.F., and Jones, D.T. (2004). The DISOPRED server for the prediction of protein disorder. Bioinformatics 20, 2138-2139.

Weinman, E.J., Hall, R.A., Friedman, P.A., Liu-Chen, L.Y., and Shenolikar, S. (2006). The association of NHERF adaptor proteins with $g$ protein-coupled receptors and receptor tyrosine kinases. Annu Rev Physiol 68, 491-505.

Wu, H., Feng, W., Chen, J., Chan, L.N., Huang, S., and Zhang, M. (2007). PDZ domains of Par-3 as potential phosphoinositide signaling integrators. Mol Cell 28, 886-898.

Yan, J., Wen, W., Xu, W., Long, J.F., Adams, M.E., Froehner, S.C., and Zhang, M. (2005). Structure of the split PH domain and distinct lipid-binding properties of the PH-PDZ supramodule of alphasyntrophin. EMBO J 24, 3985-3995. 
Yan, J., Pan, L., Chen, X., Wu, L., and Zhang, M. (2010). The structure of the harmonin/sans complex reveals an unexpected interaction mode of the two Usher syndrome proteins. Proc Natl Acad Sci U S A 107, 4040-4045.

Zhang, M., and Wang, W. (2003). Organization of signaling complexes by PDZ-domain scaffold proteins. Acc Chem Res 36, $530-538$.
Zhang, Q., Fan, J.-S., and Zhang, M. (2001). Interdomain chaperoning between PSD-95, Dlg, and Zo-1 (PDZ) domains of glutamate receptor-interacting proteins. J Biol Chem 276, 43216-43220.

Zimmermann, P., Meerschaert, K., Reekmans, G., Leenaerts, I., Small, J.V., Vandekerckhove, J., David, G., and Gettemans, J. (2002). PIP(2)-PDZ domain binding controls the association of syntenin with the plasma membrane. Mol Cell 9, 1215-1225. 